\title{
Zirconia Vs Titanium Implants - Deciding Factors
}

\author{
Saurabh Gupta* \\ Department of Oral and Maxillofacial Surgery, Academic and Research Scientist, India.
}

Received: November 02, 2016; Accepted: November 09, 2016; Published: December 05, 2016

*Corresponding author: Saurabh Gupta, Oral and Maxillofacial Surgery, Academic and Research Scientist, Bangalore,India. Axiss Dental Pvt Ltd. No 12/4 A 2, Whitefield Main Road, Opp. Hindustan Lever Ltd, Whitefield, Bengaluru, Karnataka, India, Pin: 560066. Tel: 9916203455; E-mail: saurabh. ravzz@gmail.com

\begin{abstract}
This study focuses on titanium implants in a conventional manner and the newly introduced and highly popular Zirconia implants. Some of the major areas covered here are clinical considerations and material science which includes implant materials as well as the impacts of its physical properties on outcomes of the treatment.

Titanium is the gold standard for dental implants fabrication in spite of the sensitivity and despite having unclear clinical relevance. The Zirconia implants are highly promising but further clinical studies are required. Also, there is a need of further technical experience and considerations for zirconia implants to lower the mechanical failure incidence.
\end{abstract}

\section{Introduction}

\section{Zirconia Implants Materials}

Ceramic Implant gives a distinctive esthetic solution for patients with specific needs like a thin gingival biotype and for those patients who explicitly express their need for metal-free alternative.

Post titanium dental implants usage; there have not been any report of metal reaction or allergy so far (e.g. Nickel allergy is approx 5\%). However, there are certain people who are either allergic or sensitive to other metals in their body or express concerns on having any metals inside their body [1].

For dispelling such concerns and having an implant option for such people, many firms have undertaken Research and Development on "metal-free" implants. This has resulted in making use of zirconium, a metal that is one line below titanium in the Periodic table. Use of Zirconium became widespread first in its form of crystalline (cubic form) zirconia as it resembled a flawless diamond. Several years ago single crystal sapphire implants were used; however, today they are not much heard of. In the form of zirconium oxide zro2, it is used for dental applications. Actually, it is not a form of pure zro2-there are traces of another element hafnium (Hf) and its oxide is combined with yttrium (yet another metal) to enhance its properties. This yields a white opaque product and in this form, it is labeled as ceramic, though there are metallic atoms in the material [2].
The material is very hard and strong and is also used to make bridges and crowns. While searching for alternate implant materials, it was found that zirconia also bonded to bone just like titanium.

\section{What You Should Know While Deciding Between Zirconia And Titanium Dental Implants}

Titanium Allergies:Maybe the greatest concern of patients is whether they would be allergic to titanium. Actually very rarely people get allergic to titanium. Studies have revealed very low incidence rate of $6 \%$ of patients who are allergic to titanium. Because of poor sensitivity, patch tests have restricted use and the test is confirmed for detecting sensitization in MELISA test. The test is known to yield false positives [3].

\section{Clifford Material Reactivity testing}

A blood test decides in case a blood serum sample reacts to well-known dental materials. While this test may offer valuable information regarding current allergies, outcomes cannot be as complete when it is about testing for materials which the patient is not been exposed yet.

Zirconia implants usually cannot be left to heal under the gums. Usually the osseointegration procedure takes nearly six months to complete. Once surgically placing the dental implants, they should have primary stability or a certain torque value. Implants which do not possess good primary stability must be left for healing under the gums for three to six months after placement. Majority of Zirconia dental implants may not heal under the gums due to their "one-piece" design, implying that they do not possess a removable abutment however one which is fixed to the implant.

\section{When Zirconia Is Adjusted, Micro Cracks Form And Can Cause Fractures}

The associated literature reveals that while zirconia is a "strong" material under compressive stress, it does not have adequate flexures and will fracture. This is more obvious when the material is adjusted with dental bur.

When Zirconia gets adjusted, unlike other dental materials, it 
can form micro cracks that ultimately result in fracture of crown and even of the implant.

As zirconia implants are made as one-piece design, the dentist should prepare the implant's top for customizing it to patient's mouth. This is also the case when micro cracks begin and may spread causing fracture and then the implant should have to be removed.

\section{Zirconia Implants Having A Small Diameter Are Predisposed To Fracture}

As mentioned above, zirconia is quite strong in compressive forces however rather brittle in elastic forces. Many a times in implant dentistry, a small diameter implant ranging from $3.0 \mathrm{~mm}$ $3.75 \mathrm{~mm}$ must be utilized by the dentist because of small spaces or thin bone between the teeth. A research study revealed that each implant having a diameter less than $3.75 \mathrm{~mm}$ had a disastrous fracture. Another paper revealed that out of eighteen total zirconia implants, seven implants fractured and a lost osseointegration as it might not be left for healing under the gums. It was reported by Roehling et al and Koch et al that the general rate of zirconia implants representing cracks of the implant head was nearly twenty two and thirty percent respectively. In clinical implant dentistry, such level of failure is unacceptable [4].

\section{Zirconia Implant Crowns May Usually Merely Be Cemented}

In conventional implant dentistry, it is possible to retain the dental crown either by screw or cement. Majority of zirconia implants that are FDA approved are a "one-piece design", implying they should have cemented crowns. This could not be a problem however it is. The tissues around the implants and around teeth are quite different and cement may cause numerous problems for the tissues and bone around implants [5]. In several research articles Dr. Wadhwani has proven that dental cement may get stuck into the tissues, harbors bacteria, causes bone loss and inflammation, and may even cause dental implant failure [6]. Therefore our doctors always struggle to place implants perfectly through 3D digital guided surgery for being capable of using screw retained crowns and eliminate dental cements from implant system.

\section{Full-Mouth Treatments May Not Be Completed Having One-Piece Abutments}

Several implant patients are in need of all their teeth replaced or missing all of their teeth. This is a great way or reinstating confidence, quality of life and chewing efficacy. For dental implant patients, many a times it is life changing. Such kind of treatment takes correct planning and implementation by the dentist to get superior functional and aesthetic results. Screw retained restorations and custom abutments are needed for full mouth or full arch implant treatment. Due to one-piece design of zirconia implants it would not be possible to do this [7].Advantages of Zirconia Dental Implants
Promoters of zirconia mention numerous advantages of the material:

- Dark color does not show through gums

- Zirconia does not suffer corrosion like titanium

- There are no piezoelectric current at dissimilar metal joints in mouth.

- The material is thermally non-conductive

\section{Conclusion On Zirconia Dental Implants}

Both of these materials titanium and zirconia are bio-inert and thus do not cause any inflammation and these are not rejected by body.

It is because of this reason that these are the two materials of choice for the abutment portion of the implant system. The abutment is the piece of the implant system that connects the dental crown or the tooth in the mouth to the implant that is in the jawbone. We do not need to use zirconia implants to achieve safe, predictable, and natural-looking restorations. Furthermore, we can avoid many long-term complications associated with using zirconia implants by placing titanium implants using 3D guided surgery. However, there are certain indications where zirconia implants can be used for patients that prefer zirconia over titanium.

\section{References}

1. S Berner, M Dard, J Gottlow, A Molenberg, M Wieland. Titaniumzirconium: A novel material for dental implants. Eur. Cells Mater. 2009;17(sup 1):16.

2. Zeynep Özkurt, Ender Kazazoğlu. Zirconia Dental Implants: A Literature Review, Journal of Oral Implantology. 2011;37(3):367-376. doi.org/10.1563/AAID-JOI-D-09-00079.

3. Manish Goutam, Chandu Giriyapura, Sunil Kumar Mishra, Siddharth Gupta. Titanium Allergy: A Literature Review. Indian J Dermatol. 2014;59(6): 630 .

4. Thoma DS, Benic GI, Muñoz F, Kohal R, Sanz Martin I, Cantalapiedra $\mathrm{AG}$, et all. Marginal bone-level alterations of loaded zirconia and titanium dental implants: an experimental study in the dog mandible. Clinical Oral Implants Research. 2016;27(4):412-420. doi: 10.1111/ clr.12595.

5. Rohr N, Coldea A, Zitzmann NU, Fischer J. Loading capacity of zirconia implant supported hybrid ceramic crowns. Dental Materials. 2015;31(12):279-288. doi: 10.1016/j.dental.2015.09.012.

6. Chandur P. K. Wadhwani et al. Dentistry Today, The Role of Cements in Dental Implant Success. June 1, 2012 to May 31, 2015 AGD PACE approval number: 309062

7. Brüll F, van Winkelhoff AJ, Cune MS. Zirconia dental implants: a clinical, radiographic, and microbiologic evaluation up to 3 years. Int J Oral Maxillofac Implants. 2014;29(4):914-920. doi: 10.11607/ jomi.3293. 